\title{
Classical-mechanical models without observable trajectories and the Dirac electron
}

\author{
A. A. Deriglazov* \\ Depto. de Matemática, ICE, Universidade Federal de Juiz de Fora, MG, Brazil
}

\begin{abstract}
We construct a non-Grassmann spinning-particle model which, by analogy with quantum mechanics, does not admit the notion of a trajectory within the position space. The pseudo-classical character of the model allows us to avoid the inconsistencies arising in the quantum-mechanical interpretation of a one-particle sector of the Dirac equation.
\end{abstract}

\section{INTRODUCTION}

Non-abelian gauge groups play a crucial role in field theory as well as in the Standard model. In this work we observe that they imply new possibilities when used in the construction of finite-dimensional theories as well. We suggest and discuss the notion of pseudo-classical mechanics (pCM), the term by which we refer to models with a number of observable configuration-space variables less than the number of physical degrees of freedom. In other words, we consider classical-mechanical models which, by analogy with quantum mechanics, do not admit the notion of a trajectory within the position (i.e. configuration) space. So we expect that pCM turns out to be useful in describing quantum phenomena by (semi) classical methods [1-11]. Classical mechanics with such a strange property can be constructed on the basis of a singular Lagrangian with a multi-parametric group of local symmetries. As examples of pCM we present the models which are invariant under transformation of the non-abelian gauge group with two and three local parameters. Symmetries imply functional ambiguity in solutions to equations of motion: besides the integration constants $c_{i}$, the solution depends on the arbitrary functions $e_{a}(\tau), x=f\left(\tau, c_{i}, e_{a}(\tau)\right)$. According to the general theory of singular systems [12-14], variables with ambiguous dynamics do not represent observable quantities. So, when we are dealing with the locally-invariant theory, our first task is to find candidates for observables, which are variables with unambiguous dynamics. Equivalently, we can look for the gauge-invariant variables.

We start in Section 2 with a couple of toy models and show that, generally, it is impossible to construct the observables within the position variables only. It is worth noting that on the phase space there always is the welldefined notion of a trajectory [13]. In Section 3 we consider a more realistic case, presenting the non-Grassmann model of the Dirac electron. In Section 4 we show how the pseudo-classical character of the model allows us to solve the problems arising $[2,3,18]$ when we try to apply the methods of relativistic quantum mechanics to a one-particle sector of the Dirac equation.

*Electronic address: alexei.deriglazov@ufjf.edu.br On leave of absence from Dep. Math. Phys., Tomsk Polytechnical University, Tomsk, Russia.

\section{TOY MODELS}

One of the local symmetries which will be presented in our models is reparametrization invariance. So, we first outline the reparametrization invariant formulation of a relativistic particle.

The motion of a particle in special-relativity theory can be described starting from the three-dimensional action $-m c \int d t \sqrt{c^{2}-\left(\frac{d x^{i}}{d t}\right)^{2}}$. The problem here is that the Lorentz transformations, $x^{\prime \mu}=\Lambda^{\mu}{ }_{\nu} x^{\nu}$, act on the physical dynamical variables $x^{i}(t)$ in a higher nonlinear way. To improve this, we pass from three-dimensional to four-dimensional formulation. Introducing the parametric representation $x^{\mu}(\tau)=\left(c t(\tau), x^{i}(\tau)\right)$ for the trajectory $x^{i}(t)$, the particle can be described by the Lagrangian action

$$
S=\int d \tau\left(\frac{1}{2 e}\left(\dot{x}^{\mu}\right)^{2}-\frac{e}{2} m^{2} c^{2}\right) .
$$

The corresponding Hamiltonian action reads

$$
S_{H}=\int d \tau p_{\mu} \dot{x}^{\mu}+p_{e} \dot{e}-\frac{1}{2} e\left(p^{2}+m^{2} c^{2}\right)-\lambda_{e} p_{e}
$$

where $\lambda_{e}(\tau)$ stands for the Lagrangian multiplier of the primary constraint $\pi_{e}=0$. Variation of the functional implies the Hamiltonian equations

$$
\dot{e}=\lambda_{e}, \quad \dot{p}_{e}=0, \quad \dot{x}^{\mu}=e p^{\mu}, \quad \dot{p}_{\mu}=0,
$$

as well as the constraints $\pi_{e}=0, p^{2}+m^{2} c^{2}=0$. We note that the variable $\lambda_{e}(\tau)$ cannot be determined with the constraints, nor with the dynamical equations. As a consequence (see the first of Eqs. (3)), the variable $e$ turns out to be an arbitrary function as well. Since $e(\tau)$ enters into the equation for $x^{\mu}$, its general solution contains, besides the arbitrary integration constants, the arbitrary function $e(\tau)$. Hence the only unambiguous ones among the initial variables are $p^{\mu}$ and $\pi_{e}$, see Eqs. (3). $x^{\mu}$ has one-parameter ambiguity due to $e$.

The ambiguity reflects the freedom in the choice of parametrization for the particle trajectory $(\alpha(\tau)$ is an infinitesimal function)

$$
\begin{aligned}
& \tau \rightarrow \tau^{\prime}=\tau-\alpha, \\
& x^{\mu}(\tau) \rightarrow x^{\prime \mu}\left(\tau^{\prime}\right)=x^{\mu}(\tau), \quad \text { then } \quad \delta x^{\mu}=\alpha \dot{x}^{\mu}, \\
& e(\tau) \rightarrow e^{\prime}\left(\tau^{\prime}\right)=(1+\dot{\alpha}) e(\tau) .
\end{aligned}
$$


Action (1) turns out to be invariant under the reparametrizations.

By construction, the expression for the physical trajectory $x^{i}(t)$ is obtained resolving the equation $x^{0}=x^{0}(\tau)$ with respect to $\tau, \tau=\tau\left(x^{0}\right)$, then $x^{i}(t) \equiv x^{i}\left(\tau\left(x^{0}\right)\right)$. Using the expression

$$
\frac{d f}{d x^{0}}=\frac{\dot{f}(\tau)}{\dot{x}^{0}(\tau)},
$$

for the derivative of a function given in parametric form, we obtain

$$
\frac{d x^{i}}{d t}=c \frac{\dot{x}^{i}}{\dot{x}^{0}}=c \frac{p^{i}}{\sqrt{\vec{p}^{2}+m^{2} c^{2}}},
$$

Eq. (6) coincides with that of the three-dimensional formulation. As should be the case, the physical coordinate $x^{i}(t)$ has unambiguous evolution.

Toy model which admits the position-space trajectories. Consider the Lagrangian action

$$
S=\int d \tau \frac{1}{2 e_{1}}\left(\dot{x}^{\mu}-e_{2} x^{\mu}\right)^{2} .
$$

This is written on the configuration space $x^{\mu}, e_{1}$ and $e_{2}$; the Minkowski metric is $\eta^{\mu \nu}=(-,+,+,+)$. The action is invariant under the reparametrizations, $\delta x^{\mu}=\alpha \dot{x}^{\mu}$, $\delta e_{1}=\left(\alpha e_{1}\right)^{\circ}, \delta e_{2}=\left(\alpha e_{2}\right)^{\circ}$, as well as under the following transformations with the parameter $\beta(\tau)$

$$
\delta x^{\mu}=\beta x^{\mu}, \quad \delta e_{1}=2 \beta e_{1}, \quad \delta e_{2}=\dot{\beta} .
$$

The transformations form a non-abelian group, $\left[\delta_{\alpha}, \delta_{\beta}\right]=$ $\delta_{\tilde{\beta}}, \tilde{\beta}=-\alpha \dot{\beta}$. Each local symmetry removes two degrees of freedom [22], so the number of configuration-space observables is equal to 2 .

The Hamiltonian of the theory (7) is

$$
H=\frac{e_{1}}{2} p^{2}+e_{2}(p x) .
$$

This implies the Hamiltonian equations (in what follows, we omit equations for the auxiliary variables $e_{k}, p_{e k}$, as they are not necessary for discussion of the ambiguity of the variable $x^{\mu}$ )

$$
\dot{x}^{\mu}=e_{1} p^{\mu}+e_{2} x^{\mu}, \quad \dot{p}^{\mu}=-e_{2} p^{\mu},
$$

as well as the constraints

$$
p^{2}=0, \quad(p x)=0 .
$$

The equation for $x^{\mu}$ has two-parametric ambiguity due to $e_{1}$ and $e_{2}$, while that for $p^{\mu}$ has one-parametric ambiguity. Inside the light-cone, we construct the variables

$$
\tilde{x}^{\mu}=\frac{x^{\mu}}{\sqrt{-x^{2}}}, \quad \tilde{p}^{\mu}=\sqrt{-x^{2}} p^{\mu} .
$$

Their equations read

$$
\dot{\tilde{x}}^{\mu}=e_{1} \frac{p^{\mu}}{\sqrt{-x^{2}}}, \quad \dot{\tilde{p}}^{\mu}=0 .
$$

They can be compared with Eqs. (3). The constraints (11) acquire the form $\tilde{p}^{2}=0,(\tilde{p} \tilde{x})=0$. Note that $\tilde{x}$ is $\beta$-invariant variable. So the ambiguity presented in Eq. (13) is due to the reparametrization symmetry. In accordance with this observation, we assume that the functions $\tilde{x}^{\mu}(\tau)$ and $\tilde{p}^{\mu}(\tau)$ represent the reparametrizationinvariant variables $\tilde{x}^{i}(t)$ and $\tilde{p}^{\mu}(t)$ in the parametric form. Their equations of motion read

$$
\frac{d \tilde{x}^{i}}{d t}=c \frac{p^{i}}{p^{0}} \equiv c \frac{\tilde{p}^{i}}{\tilde{p}^{0}}, \quad \frac{d \tilde{p}^{\mu}}{d t}=0 .
$$

Since they are unambiguous, the variables $\tilde{x}^{i}(t)$ and $\tilde{p}^{\mu}(t)$ are candidates for the observables.

By construction, $\tilde{x}^{\mu}$ obey the identity $\tilde{x}^{\mu} \tilde{x}_{\mu}=-1$. So only three of them can be taken as coordinates of the configuration space. Adding the variable $\sigma=\frac{1}{\sqrt{-x^{2}}}$ to the set $\tilde{x}^{i}$, we obtain a coordinate system. As the two independent observables we can take the gauge-invariant variables $\tilde{x}^{1}(t)$ and $\tilde{x}^{2}(t)$. Hence, the present model admits observable trajectories constructed within the position space; see Eq. (12).

To conclude, we point out that Poisson brackets of the Lorentz-covariant observables (12) generate the noncommutative algebra

$$
\left\{\tilde{x}^{\mu}, \tilde{p}^{\nu}\right\}=N^{\mu \nu}(\tilde{x}),\left\{\tilde{x}^{\mu}, \tilde{x}^{\nu}\right\}=0,\left\{\tilde{p}^{\mu}, \tilde{p}^{\nu}\right\}=\tilde{p}^{[\mu} \tilde{x}^{\nu]}
$$

Here and below we denote

$$
N^{\mu \nu}(a) \equiv \eta^{\mu \nu}-\frac{a^{\mu} a^{\nu}}{a^{2}} .
$$

Toy model without position-space trajectories. Consider the following Lagrangian action written for the variables $x^{\mu}, \omega^{\mu}, e_{1}$ and $e_{2}$

$$
\begin{gathered}
S=\int d \tau \frac{1}{2\left(e_{1}-e_{2}^{2}\right)}\left[(D x)^{2}+2 e_{2}(D x \dot{\omega})+e_{1} \dot{\omega}^{2}\right]- \\
\frac{e_{1}}{2} m^{2} c^{2}+\frac{1}{2} \omega^{2} .
\end{gathered}
$$

We have denoted $D x^{\mu} \equiv \dot{x}^{\mu}-e_{2} \omega^{\mu}$. The non-abelian gauge group is composed by reparametrizations as well as by the following transformations with the parameter $\beta(\tau)$ :

$$
\delta x^{\mu}=\frac{\beta}{e_{1}-e_{2}^{2}}\left(D x^{\mu}+e_{2} \dot{\omega}^{\mu}\right), \quad \delta e_{1}=\dot{\beta} .
$$

This implies that the number of physical degrees of freedom on configuration (phase) space is equal to 6 (12).

Denoting conjugate momenta of $x, \omega$ by $p, \pi$, the Hamiltonian of the theory (17) reads

$$
H=\frac{1}{2} \pi^{2}-\frac{1}{2} \omega^{2}+\frac{1}{2} e_{1}\left(p^{2}+m^{2} c^{2}\right)+e_{2} p_{\mu}\left(\omega^{\mu}-\pi^{\mu}\right)
$$

This implies the Hamiltonian equations

$$
\dot{x}^{\mu}=e_{1} p^{\mu}+e_{2}\left(\omega^{\mu}-\pi^{\mu}\right), \quad \dot{p}^{\mu}=0,
$$




$$
\dot{\omega}^{\mu}=\pi^{\mu}-e_{2} p^{\mu}, \quad \dot{\pi}^{\mu}=\omega^{\mu}-e_{2} p^{\mu} .
$$

as well as the first-class constraints

$$
p^{2}+m^{2} c^{2}=0, \quad(p, \omega-\pi)=0 .
$$

The equation for $x$ has two-parametric ambiguity due to $e_{1}$ and $e_{2}$, while those for $\omega$ and $\pi$ have one-parametric ambiguity.

Taking into account the first-class constraints, we could expect 6 observable dynamical variables on the configuration space. However, it is easy to see that any configuration-space quantity $a^{\mu}(x, \omega)$ with oneparametric ambiguity is proportional to $\omega^{\mu}$. Similarly to the previous model, this can be used to construct only three unambiguous dynamic variables. As the sixdimensional configuration space can not be spanned with the unambiguous variables, the model represents an example of pseudo-classical mechanics.

On the phase space we can construct various variables with one-parametric ambiguity due to $e_{2}$

$$
\begin{gathered}
\tilde{x}^{\mu}=x^{\mu}-\frac{(p x)}{p^{2}} p^{\mu}, \quad \dot{\tilde{x}}^{\mu}=e_{2}\left(\omega^{\mu}-\pi^{\mu}\right), \\
\tilde{p}^{\mu}=p^{\mu}, \quad \dot{\tilde{p}}^{\mu}=0, \\
\tilde{\omega}^{\mu}=\frac{(\omega+\pi, p)}{2 p^{2}}\left(\omega^{\mu}-\pi^{\mu}\right), \quad \dot{\tilde{\omega}}^{\mu}=-e_{2}\left(\omega^{\mu}-\pi^{\mu}\right), \\
\tilde{\pi}^{\mu}=\frac{\omega^{\mu}+\pi^{\mu}}{\sqrt{(\omega+\pi)^{2}}}, \quad \dot{\tilde{\pi}}^{\mu}=-\frac{2 e_{2} N^{\mu \nu}(\omega+\pi) p_{\nu}}{\sqrt{(\omega+\pi)^{2}}} . \\
J^{\mu \nu}=\omega^{\mu} \pi^{\nu}-\omega^{\nu} \pi^{\mu}, \quad \dot{j}^{\mu \nu}=e_{2} p^{[\mu}(\omega-\pi)^{\nu]}
\end{gathered}
$$

The constraints (21) acquire the form $\tilde{p}^{2}+m^{2} c^{2}=0$, $(\tilde{p} \tilde{\omega})=0$. The new variables are invariants of the $\beta$ transformation ${ }^{1}$. So the ambiguity presented in Eqs. (22)-(26) is due to the reparametrization symmetry. Similarly to the case of the relativistic particle, we assume that the functions $\tilde{x}^{\mu}(\tau), \tilde{p}^{\mu}(\tau), \tilde{\omega}^{\mu}(\tau), \tilde{\pi}^{\mu}(\tau)$ and $J^{\mu \nu}(\tau)$ represent the physical variables $\tilde{x}^{i}(t), \tilde{p}^{\mu}(t), \ldots$ in the parametric form. According to Eq. (5), the dynamics of the physical variables is unambiguous.

The Poisson-bracket algebra of the Lorentz-covariant observables is highly noncommutative; the nonvanishing brackets are

$$
\begin{array}{r}
\left\{\tilde{x}^{\mu}, \tilde{x}^{\nu}\right\}=\frac{p^{[\mu} \tilde{x}^{\nu]}}{p^{2}}, \quad\left\{\tilde{x}^{\mu}, p^{\nu}\right\}=N^{\mu \nu}(p), \\
\left\{\tilde{\omega}^{\mu}, \tilde{\omega}^{\nu}\right\}=\frac{p^{[\mu} \tilde{\omega}^{\nu]}}{p^{2}}, \quad\left\{\tilde{\omega}^{\mu}, \tilde{\pi}^{\nu}\right\}=\frac{(p \tilde{\pi})}{p^{2}} N^{\mu \nu}(\tilde{\pi}),
\end{array}
$$

\footnotetext{
${ }^{1}$ Due to the identities $\tilde{x}^{\mu} \tilde{p}_{\mu}=0, \tilde{\pi}^{\mu} \tilde{\pi}_{\mu}=1$ and $\epsilon^{i j k} J_{i j} J_{0 k}=0$, not all of them are independent.
}

$$
\begin{gathered}
\left\{\tilde{x}^{\mu}, \tilde{\omega}^{\nu}\right\}=\frac{\tilde{\pi}^{\mu} \tilde{\omega}^{\nu}}{(p \tilde{\pi})}-\frac{p^{\mu} \tilde{\omega}^{\nu}}{p^{2}}, \\
\left\{J^{\mu \nu}, J^{\alpha \beta}\right\}=\eta^{\mu \alpha} J^{\nu \beta}-\eta^{\mu \beta} J^{\nu \alpha} \\
-\eta^{\nu \alpha} J^{\mu \beta}+\eta^{\nu \beta} J^{\mu \alpha} \\
\left\{J^{\mu \nu}, \tilde{\omega}^{\alpha}\right\}=\eta^{\alpha[\mu} \tilde{\omega}^{\nu]}+\frac{p^{[\mu} \tilde{\pi}^{\nu]} \tilde{\omega}^{\alpha}}{(p \tilde{\pi})}, \\
\left\{J^{\mu \nu}, \tilde{\pi}^{\alpha}\right\}=\eta^{\alpha[\mu} \tilde{\pi}^{\nu]} .
\end{gathered}
$$

The set of 12 independent observables of the phasespace can be selected as follows. We parameterize the initial space by the coordinates $x^{0}, \tilde{x}^{i}, \tilde{p}^{\mu}, \tilde{\omega}^{\mu}, S^{i} \equiv \epsilon^{i j k} J_{j k}$ and $\gamma=\sqrt{(\omega+\pi)^{2}}$. The dynamics of the theory is restricted on the surface $\tilde{p}^{2}+m^{2}=0,(\tilde{p} \tilde{\omega})=0$ which is invariant under the action of the gauge group ${ }^{2}$. The surface can be parameterized by $x^{0}, \tilde{x}^{i}, \tilde{p}^{i}, \tilde{\omega}^{i}, S^{i}$ and $\gamma$. The corresponding dynamic variables $\tilde{x}^{i}(t), \tilde{p}^{i}(t), \tilde{\omega}^{i}(t)$ and $S^{i}(t)$ have unambiguous dynamics. Hence we can take them as the independent observables.

\section{NON-GRASSMANN MECHANICAL MODEL OF THE DIRAC ELECTRON}

As a more realistic example of pCM, we discuss the spinning-particle model suggested in a recent work [15]. The configuration space of the model consist of the dynamical variables $Q^{\alpha}(\tau)=\left(x^{\mu}, \omega^{\nu}, \omega^{5}\right)$ as well as the auxiliary variables $e_{l}, l=1,2,3,4 . x^{\mu}$ are coordinates of the Minkowski space with the metric $\eta^{\mu \nu}=(-,+,+,+)$. The spin-space $\omega^{A}=\left(\omega^{\mu}, \omega^{5}\right)$ is equipped with $S O(2,3)$ metric $\eta^{A B}=(-,+,+,+,-)$. Consider the Poincareinvariant Lagrangian

$$
L=\frac{1}{2} G_{\alpha \beta} \dot{Q}^{\alpha} \dot{Q}^{\beta}-\frac{e_{4}}{2} \omega^{A} \omega_{A}-\frac{e_{l}}{2} a_{l} .
$$

We have denoted $a_{1}=m^{2} c^{2}, a_{2}=m c \hbar$, and $a_{3}, a_{4}$ are real numbers. In what follows, we discuss the free theory. Interaction with an external electromagnetic field will be discussed at the end of Section 4. The kinetic term looks like that of a free particle moving on the curved ninedimensional space with the metric

$$
G_{\alpha \beta}=\left(\begin{array}{ccc}
e_{3} G_{\mu \nu} & -e_{2} \omega^{5} G_{\mu \nu} & \frac{e_{2}}{A} \omega_{\mu} \\
-e_{2} \omega^{5} G_{\mu \nu} & e_{1} G_{\mu \nu}+\frac{e_{2}^{2} \omega_{\mu} \omega_{\nu}}{e_{3} A} & -\frac{e_{2}^{2} \omega^{5}}{e_{3} A} \omega_{\mu} \\
\frac{e_{2}}{A} \omega_{\nu} & -\frac{e_{2}^{2} \omega^{5}}{e_{3} A} \omega_{\nu} & -\frac{B}{e_{3} A}
\end{array}\right)
$$

We have denoted $G_{\mu \nu}=\frac{1}{B}\left[\eta_{\mu \nu}-\frac{e_{2}^{2} \omega_{\mu} \omega_{\nu}}{A}\right], B=e_{1} e_{3}-$ $e_{2}^{2}\left(\omega^{5}\right)^{2}$ and $A=B+e_{2}^{2}\left(\omega^{\mu}\right)^{2}$.

We introduce the abbreviation

$$
D x^{\mu} \equiv \dot{x}^{\mu}-\frac{e_{2}}{e_{3}}\left(\omega^{5} \dot{\omega}^{\mu}-\omega^{\mu} \dot{\omega}^{5}\right),
$$

\footnotetext{
${ }^{2}$ We use the phase-space form of reparametrizations, $\delta \tilde{\omega}^{\mu}=$ $-\alpha e_{2}\left(\omega^{\mu}-\pi^{\mu}\right), \delta p^{\mu}=0$; see [15] for details.
} 
then the Lagrangian (28) can be written as follows,

$$
L=\frac{e_{3}}{2} G_{\mu \nu} D x^{\mu} D x^{\nu}+\frac{1}{2 e_{3}} \dot{\omega}^{A} \dot{\omega}_{A}-\frac{e_{4}}{2} \omega^{A} \omega_{A}-\frac{e_{l}}{2} a_{l} .(31)
$$

The Lagrangian is invariant under a three-parametric group of local symmetries. One of them is the reparametrization symmetry. Besides, there are two more symmetries with the local parameters $\beta(\tau), \gamma(\tau)$

$$
\begin{gathered}
\delta_{\beta} x^{\mu}=\beta p^{\mu}, \quad \delta_{\beta} e_{1}=\dot{\beta} \\
\delta_{\gamma} \omega^{A}=\gamma e_{3} \pi^{A}, \quad \delta_{\gamma} \pi^{A}=-\gamma e_{4} \omega^{A}, \\
\delta_{\gamma} e_{3}=\left(\gamma e_{3}\right), \quad \delta_{\gamma} e_{4}=\left(\gamma e_{4}\right) .
\end{gathered}
$$

Here $p_{\mu}=\frac{\partial L}{\partial \dot{x}^{\mu}}, \pi_{A}=\frac{\partial L}{\partial \dot{\omega}^{A}}$. In the Hamiltonian formulation, the Lagrangian (31) leads to the following Hamiltonian $[15]$

$$
\begin{gathered}
H=\frac{e_{1}}{2}\left(p^{2}+m^{2} c^{2}\right)+\frac{e_{2}}{2}\left(p_{\mu} J^{5 \mu}+m c \hbar\right)+ \\
\frac{e_{3}}{2}\left(\pi^{A} \pi_{A}+a_{3}\right)+\frac{e_{4}}{2}\left(\omega^{A} \omega_{A}+a_{4}\right)+\lambda_{e a} \pi_{e a},
\end{gathered}
$$

where

$$
J^{5 \mu}=2\left(\omega^{5} \pi^{\mu}-\omega^{\mu} \pi^{5}\right)
$$

If we omit the spin-space coordinates, $\omega^{A}=\pi_{A}=0$, the Hamiltonian reduces to that of the spinless particle, see (2).

The Hamiltonian implies the constraints

$$
\begin{gathered}
\omega^{A} \omega_{A}+a_{4}=0, \quad \pi^{A} \omega_{A}=0 ; \\
p^{2}+m^{2} c^{2}=0, \quad \pi^{A} \pi_{A}+a_{3}=0 ; \\
p_{\mu} J^{5 \mu}+m c \hbar=0 .
\end{gathered}
$$

The first one states that configuration space of spin is anti-de Sitter space. The constraints (36) form the second-class pair while those of Eqs. (37) and (38) are the first-class constraints. The constraint (38), being imposed on the state vector, leads to the Dirac equation ${ }^{3}$, $\left(\gamma^{\mu} \hat{p}_{\mu}+m c\right) \Psi=0$ (see [15] for details).

The Hamiltonian equations of the theory read

$$
\begin{aligned}
\dot{x}^{\mu} & =e_{1} p^{\mu}+\frac{1}{2} e_{2} J^{5 \mu}, & \dot{p}^{\mu} & =0 ; \\
\dot{\omega}^{\mu} & =e_{3} \pi^{\mu}+e_{2} \omega^{5} p^{\mu}, & \dot{\pi}^{\mu} & =e_{2} \pi^{5} p^{\mu}-\frac{a_{3}}{a_{4}} e_{3} \omega^{\mu} ;
\end{aligned}
$$

\footnotetext{
3 The present model implies both the Dirac equation and the massshell condition $p^{2}+m^{2} c^{2}=0$. The model without the massshell condition has been discussed in $[16,17]$. This shows the same undesirable properties as those of the Dirac equation in the classical limit $[2,3]$.
}

$$
\dot{\omega}^{5}=e_{3} \pi^{5}+e_{2}(p \omega), \quad \dot{\pi}^{5}=e_{2}(p \pi)-\frac{a_{3}}{a_{4}} e_{3} \omega^{5} .
$$

The only unambiguous variable is $p^{\mu}$. The configuration space variables $x^{\mu}, \omega^{A}$ have two-parametric ambiguity.

Let us compute the total number of physical degrees of freedom. Omitting the auxiliary variables and the corresponding constraints, we have 18 phase-space variables $x^{\mu}, p_{\mu}, \omega^{A}, \pi_{A}$ subject to the constraints (36)- (38). Taking into account that each second-class constraint rules out one variable, whereas each first-class constraint rules out two variables, the number of physical degrees of freedom on the phase space is $18-(2+2 \times 3)=10$. Hence we could expect five observables on the configuration space. However, using the configuration-space variables only, it is impossible to construct five unambiguous quantities. Thus, once again we have an example of pseudo-classical mechanics.

Let us discuss the physical sector of the phase space. A brief inspection of the equations of motion allows us to construct the Lorentz-covariant variables with oneparameter ambiguity. They are the five-dimensional angular-momentum tensor ${ }^{4} J^{A B}=2 \omega^{[A} \pi^{B]}$; this obeys

$$
\dot{J}^{5 \mu}=-e_{2} J^{\mu \nu} p_{\nu}, \quad \dot{J}^{\mu \nu}=-e_{2} J^{5[\mu} p^{\nu]}
$$

as well as the position variable

$$
\tilde{x}^{\mu}=x^{\mu}+\frac{1}{2 p^{2}} J^{\mu \nu} p_{\nu}, \quad \dot{\tilde{x}}^{\mu}=\tilde{e} p^{\mu} .
$$

We have denoted $\tilde{e} \equiv e_{1}+\frac{\hbar e_{2}}{2 m c}$. So the reparametrizationinvariant variable $\tilde{x}^{i}(t)$ has a deterministic evolution:

$$
\frac{d \tilde{x}^{i}}{d t}=c \frac{\dot{\tilde{x}}^{i}}{\dot{\tilde{x}}^{0}}=c \frac{p^{i}}{p^{0}}
$$

As the classical four-dimensional spin vector, we take the Pauli-Lubanski vector which has no precession in the free theory

$$
S^{\mu}=\frac{1}{2} \epsilon^{\mu \nu \alpha \beta} p_{\nu} J_{\alpha \beta}, \quad \dot{S}^{\mu}=0
$$

In the rest frame $p^{\mu}=(m c, 0,0,0)$, it reduces to the three-dimensional rotation generator, $S^{0}=0, S^{i}=$ $\frac{1}{2} m c \epsilon^{i j k} S_{j k}$, as is expected in the non-relativistic limit.

We point out that the second term in Eq. (41) has the structure typical for non-commutative extensions of the usual mechanics, see [23].

\footnotetext{
${ }^{4}$ Note that the constraints (36) and (37) fix the value of the Casimir operators of the $S O(2,3)$ group. Besides, they guarantee that $J^{5 \mu}$ is the time-like vector $\left(J^{5 \mu}\right)^{2}=-4\left(a_{3}\left(\omega^{5}\right)^{2}+\right.$ $\left.a_{4}\left(\pi^{5}\right)^{2}\right)<0$, for positive values of $a_{3}, a_{4}$; see [15] for details.
} 


\section{CONCLUSION. PSEUDO-CLASSICAL MECHANICS AND THE CLASSICAL LIMIT OF THE DIRAC EQUATION}

Although a true understanding of spin is achieved in the framework of quantum electrodynamics, a lot of effort has been spent in attempts to construct a mechanical model of a spinning electron, see [1-11, 1517] and references therein. The Dirac spinor $\Psi$ can be used to construct the four-dimensional current vector, $\bar{\Psi} \gamma^{\mu} \Psi$, which preserves for solutions to the Dirac equation, $\partial_{\mu}\left(\bar{\Psi} \gamma^{\mu} \Psi\right)=0$. Hence its null-component, $\Psi^{\dagger} \Psi \geq 0$, admits the probabilistic interpretation, and we expect that a one-particle sector of the Dirac equation could be described in the framework of relativistic quantum mechanics (RQM).

However, it is well known that adopting the RQM interpretation, we arrive at a rather strange and controversial picture $[2,3,18]$. To recall this to mind, we use the Dirac matrices $\alpha^{i}$ and $\beta$, to represent the Dirac equation in the form of the Schrödinger one

$$
i \hbar \partial_{t} \Psi=\hat{H} \Psi, \quad \hat{H}=c \alpha^{i} \hat{p}_{i}+m c^{2} \beta .
$$

Then $\hat{H}$ may be interpreted as the Hamiltonian. If we pass from the Schrödinger to the Heisenberg picture, the time derivative of an operator $a$ is $i \hbar \dot{a}=[a, H]$. For the basic operators of the Dirac theory we obtain

$$
\dot{x}_{i}=c \alpha_{i}, \quad i \hbar \dot{\alpha}_{i}=2\left(c p_{i}-H \alpha_{i}\right), \quad \dot{p}_{i}=0
$$

Below we enumerate the inconsistences arising in the RQM interpretation of these equations and show how our pCM allow us to avoid them.

The wrong balance of the number of degrees of freedom. Assuming $x$ as the position operator, the first equation in (45) implies that the operator $c \alpha^{i}$ represents the velocity of the particle. Then the physical meaning of the operator $p^{i}$ became rather obscure in both the semiclassical and the RQM framework. Using the quantum-fieldtheory arguments, Foldy and Wouthuysen [6] argued that the basic operator $x$ which appears in the Dirac equation does not correspond to the observable quantity. Then they constructed the position operator $\mathbf{X}^{i}$ with reasonable properties.

Our model supports the Foldy-Wouthuysen suggestion. Indeed, we observe that the variable $x^{\mu}$ is not a gaugeinvariant quantity in our model, so it is not an observable. The observable variable which can be associated with the particle position is $\tilde{x}^{i}(t)$. According to Eq. (42), $p^{i}$ determines its velocity. $\tilde{x}^{\mu}(\tau)$ written in Eq. (41) represents the Lorentz-covariant analog of the operator $\mathbf{X}^{i}$ in the classical theory. We also point out that the FoldyWouthuysen transition $x \rightarrow \mathbf{X}$ corresponds in pCM to the transition from the gauge-non-invariant to the gaugeinvariant variable.

Zitterbewegung. The equations (45) can be solved, with the result for $x^{i}(t)$ being $[2,3] x^{i}=a^{i}+b p^{i} t+$ $c^{i} \exp \left(-\frac{2 i H}{\hbar} t\right)$. The last term on the r.h.s. of this equation states that the free electron experiences rapid oscillations with higher frequency $\frac{2 H}{\hbar} \sim \frac{2 m c^{2}}{\hbar}$. It is often assumed that Zitterbewegung represents the physically observable motion of a real particle [18]. The analogous systems that are described by a Dirac-type equation and simulate Zitterbewegung are under intensive study in different physical set-ups, including graphene, trapped ions, photonic lattices and ultracold atoms, see [19] and references therein.

Our model excludes the Zitterbewegung, since this represents the dynamics of the unobservable variable $x^{\mu}$. The observable variable $\tilde{x}^{i}$ moves along a straight line, see Eq. (42).

Velocity of an electron. Since the velocity operator $c \alpha^{i}$ has eigenvalues $\pm c$, we conclude that a measurement of a component of the velocity of a free electron is certain to lead to the result $\pm c$.

In our model, the conjugate momentum $p^{\mu}$ determines the velocity of the physical coordinate $\tilde{x}^{\mu}$, see Eqs. (41), (42). Then the mass-shell condition (37) guarantees that the particle cannot exceed the speed of light.

Bargmann-Michel-Telegdi (BMT) equations. In their seminal work [7], Bargmann, Michel and Telegdi suggested relativistic equations for the classical trajectories and spin precession in uniform fields. The equations practically exactly reproduced the spin dynamics of polarized beams and agreed with the calculations based on the Dirac theory. While the BMT model apparently does not imply the Dirac equation, there is a certain relationship between the two schemes. Namely, the first term of the WKB solution to the Dirac equation can be used to construct the quantities which obey the BMT equations [20, 21].

We have seen above that performing the canonical quantization of our model in the initial variables we arrive at the Dirac equation. Now we show that physical variables of the model obey the BMT equations.

We take the Hamiltonian of interacting theory in the form

$$
\begin{gathered}
H=\frac{e_{1}}{2}\left(\mathcal{P}^{2}+\frac{e}{2 c} F_{\mu \nu} J^{\mu \nu}+m^{2} c^{2}\right)+ \\
\frac{e_{2}}{2}\left(\mathcal{P}_{\mu} J^{5 \mu}+m c \hbar\right)+ \\
\frac{e_{3}}{2}\left(\pi^{A} \pi_{A}+a_{3}\right)+\frac{e_{4}}{2}\left(\omega^{A} \omega_{A}+a_{4}\right)+\lambda_{e a} \pi_{e a},
\end{gathered}
$$

where $\mathcal{P}_{\mu}=p_{\mu}+\frac{e}{c} A_{\mu}$ is the mechanical momentum. This does not break the local symmetries presented in the model for the case of uniform electric and magnetic fields. However, the interaction deforms the physical sector: unambiguous variables of the free theory no longer remain unambiguous in the interacting theory. In particular, $\tilde{x}^{\mu}, p^{\mu}$ and $S^{\mu}$ now have two-parametric ambiguity. Up to the order $O\left(\hbar^{2}\right)$, the quantities with one-parametric ambiguity turn out to be

$$
\mathbf{P}_{\mu}=\mathcal{P}^{\mu}-\frac{e}{2 c \mathcal{P}^{2}}(F J \mathcal{P})_{\mu}, \quad \dot{\mathbf{P}}_{\mu}=-\frac{e}{c} \tilde{e} F_{\mu \nu} \mathbf{P}^{\nu}
$$




$$
\begin{array}{rlrl}
\mathbf{x}^{\mu} & =x^{\mu}+\frac{1}{2 \mathbf{P}^{2}} J^{\mu \nu} \mathbf{P}_{\nu}, & & \dot{\mathbf{x}}^{\mu}=\tilde{e} \mathbf{P}^{\mu} \\
\mathbf{S}^{\mu}=\frac{1}{2} \epsilon^{\mu \nu \alpha \beta} \mathbf{P}_{\nu} J_{\alpha \beta}, & \dot{\mathbf{S}}^{\mu}=-\frac{e}{c} \tilde{e} F^{\mu}{ }_{\nu} \mathbf{S}^{\nu} .
\end{array}
$$

We have denoted $\tilde{e}=e_{1}-\frac{m c \hbar}{2 \mathcal{P}^{2}} e_{2}$. The corresponding reparametrization-invariant variables obey the BMT equations with $g=2$

$$
\frac{d}{d t} \mathbf{x}^{i}=c \frac{\mathbf{P}^{i}}{\mathbf{P}^{0}}, \quad \frac{d}{d t} \mathbf{P}^{i}=-\frac{e}{\mathbf{P}^{0}} F^{i \nu} \mathbf{P}_{\nu}
$$

[1] J. Frenkel, Z. fur Physik 37, (1926) 243.

[2] E. Schrödinger, Sitzunger. Preuss. Akad. Wiss. Phys.Math. Kl. 24 (1930) 418.

[3] P. A. M. Dirac, The Principles of Quantum Mechanics (Clarendon Press, Oxford, 1958) p. 261.

[4] M. H. L. Pryce, Proc. Roy. Soc. A 195 (1948) 62.

[5] T. D. Newton and E. P. Wigner, Rev. Mod. Phys. 21 (1949) 400.

[6] L. L. Foldy and S. A. Wouthuysen, Phys. Rev. 78 (1950) 29.

[7] V. Bargmann, L. Michel and V.L. Telegdi, Phys. Rev. Lett. 2 (1959) 435.

[8] A. J. Hanson and T. Regge, Ann. Phys. 87 (1974) 498.

[9] F. A. Berezin and M. S. Marinov, JETP Lett 21 (1975) 320; Ann. Phys. 104 (1977) 336.

[10] A. Bermudez, M. A. Martin-Delgado, A. Luis, Phys. Rev. A 77 (2008) 063815.

[11] P. Garbaczewski, Phys. Lett. A 73 (1979) 280.

[12] P. A. M. Dirac, Can. J. Math. 2 (1950) 129; P. A. M. Dirac, Lectures on Quantum Mechanics (Yeshiva University, New York, 1964).

[13] D. M. Gitman and I. V. Tyutin, Quantization of Fields

$$
\frac{d}{d t} \mathbf{S}^{\mu}=-\frac{e}{\mathbf{P}^{0}} F^{\mu \nu} \mathbf{S}_{\nu}
$$

\section{ACKNOWLEDGMENTS}

This work has been supported by the Brazilian foundation FAPEMIG.

with Constraints (Springer-Verlag, Berlin, 1990) p. 36.

[14] A. A. Deriglazov, Classical Mechanics, Hamiltonian and Lagrangian Formalism (Springer-Verlag, Berlin Heidelberg, 2010).

[15] A. A. Deriglazov, Phys. Lett. A 376 (2012) 309; arXiv: 1106.5228

[16] A. A. Deriglazov, Ann. Phys. 327 (2012) 398, arXiv: 1107.0273

[17] A. A. Deriglazov, B. F. Rizzuti, G. P. Z. Chauca, P. S. Castro, Non-Grassmann mechanical model of the Dirac equation, arXiv:1202.5757.

[18] J. J. Sakurai, Advanced Quantum Mechanics, (AddisonWesley Publishing Company Inc., 1967) p. 112, 139.

[19] F. Zahringer, G. Kirchmair, R. Gerritsma, E. Solano, R. Blatt, and C. F. Roos, Nature (London) 463, 68 (2010);

[20] S. I. Rubinow and J. B. Keller, Phys. Rev. 131 (1963) 2789.

[21] K. Rafanelli and R. Schiller, Phys. Rev. 135 (1964) B279.

[22] A. A. Deriglazov, J. Phys. A 40 (2007) 11083.

[23] A. A. Deriglazov, Phys. Lett. B 530 (2002), 235, Phys. Lett. B 555 (2003), 83; J. High Energy Phys. 3 (2003) 021. 